\title{
Warm absorber, reflection and Fe K line in the X-ray spectrum of IC 4329A
}

\author{
M. Cappi ${ }^{1}$, T. Mihara ${ }^{1}$, M. Matsuoka ${ }^{1}$, K. Hayashida ${ }^{2}$, \\ K.A. Weaver ${ }^{3,4}$ and C. Otani ${ }^{1}$ \\ 1 The Institute of Physical and Chemical Research (RIKEN), 2-1, Hirosawa, Wako, \\ Saitama 351-01, Japan \\ ${ }^{2}$ Department of Physics, Faculty of Science, University of Osaka, 1-1 Machikaneyama- \\ chou, Toyonaka, Osaka 560 \\ 3 Department of Astronomy and Astrophysics, 525 Davey Lab., Pennsylvania State \\ University, University Park, PA 16802 \\ ${ }^{4}$ Presently at The Johns Hopkins University, Department of Physics and Astronomy, \\ 3400 N. Charles Street, Baltimore, MD 21218
}




\begin{abstract}
Results from the X-ray spectral analysis of the ASCA PV phase observation of the Seyfert 1 galaxy IC 4329A are presented. We find that the 0.4 - $10 \mathrm{keV}$ spectrum of IC 4329A is best described by the sum of a steep $(\Gamma \sim 1.98)$ power-law spectrum passing through a warm absorber plus a strong reflection component and associated Fe K line, confirming recent results (Madejski et al. 1995, Mushotsky et al. 1995). Further cold absorption in excess of the Galactic value and covering the entire source is also required by the data, consistent with the edge-on galactic disk and previous X-ray measurements. The effect of the warm absorber at soft X-ray energies is best parameterized by two absorption edges, one consistent with OVI, OVII or NVII, the other consistent with OVIII. A description of the soft excess in terms of blackbody emission, as observed in some other Seyfert 1 galaxies, is ruled out by the data. A large amount of reflection is detected in both the GIS and SIS detectors, at similar intensities. We find a strong correlation between the amount of reflection and the photon index, but argue that the best solution with the present data is that given by the best statistical fit. The model dependence of the Fe $\mathrm{K}$ line parameters is also discussed. Our best fit gives a slightly broad $(\sigma \simeq 0.11 \pm 0.08 \mathrm{keV})$ and redshifted $(\mathrm{E} \simeq 6.20 \pm 0.07 \mathrm{keV}) \mathrm{Fe}$ $\mathrm{K}$ line, with equivalent width $\simeq 89 \pm 33 \mathrm{eV}$. The presence of a weak Fe $\mathrm{K}$ line with a strong reflection can be reconciled if one assumes iron underabundances or ionized reflection. We also have modeled the line with a theoretical line profile produced by an accretion disk. This yields results in better agreement with the constraints obtained from the reflection component.
\end{abstract}

Key-words: galaxies: individual (IC 4329A) - galaxies: Seyfert - X-rays: galaxies 


\section{Introduction}

X-ray observations of Seyfert 1 galaxies strongly support the hypothesis that optically thick (column density $\gtrsim 10^{23} \mathrm{~cm}^{-2}$ ) matter surrounds the innermost regions of Active Galactic Nuclei (AGN) (Nandra \& Pounds 1994). The detection of a bump in the continuum emission above $\sim 7 \mathrm{keV}$ and an iron fluorescence line at $\sim 6.4 \mathrm{keV}$ in several Seyfert galaxies are compelling evidence that the intrinsic continuum is reprocessed by cold matter (Guilbert \& Rees 1988, Lightman \& White 1988). Recent ASCA observations also confirm the existence of ionized matter (the so-called warm absorber) along the line of sight of some Seyfert 1 galaxies (e.g., MCG-6-30-15: Fabian et al. 1994; NGC3227: Ptak et al. 1994; NGC3783: George, Turner \& Netzer 1995). In these sources, the warm absorber alone can explain both the absorption features detected around $1 \mathrm{keV}$ and the soft-excess emission. However, this seems not to be the rule for all Seyfert 1 galaxies (Matsuoka 1994). Some of them, like NGC7469, do not show the presence of ionized absorption but require extra soft emission best characterized by a low temperature blackbody (Guainazzi et al. 1994). Others, like NGC4051 and Mkn766, require both the warm absorber and blackbody component (Mihara et al. 1994, Leighly et al. 1995, Guainazzi et al., in preparation).

In light of these general considerations, the ASCA observation of the bright Seyfert 1 galaxy IC 4329A ( $\mathrm{z}=0.016$, Wilson \& Penston 1979) provides new insights on these issues. From the Ginga observation in the 1.7 - $30 \mathrm{keV}$ energy band, IC 4329A is known to exhibit a strong reflection component which flattens the 1.7 - $30 \mathrm{keV}$ photon index from an intrinsic value of $1.97 \pm 0.04$ to the apparent value of $1.71 \pm 0.01$ (Miyoshi et al. 1988, Piro, Yamauchi \& Matsuoka 1990, hereafter PYM90). The reflection component is accompanied by an iron fluoresence line at $\mathrm{E} \sim 6.2 \mathrm{keV}$, with an equivalent width $(\mathrm{EW})$ of $\simeq 100 \pm 20 \mathrm{eV}$. Evidence of day to day $(\sim 50 \%)$ variations in the amount of reflection relative to the direct continuum have also been reported (Fiore et al. 1992). In the ROSAT soft energy band (0.1-2.4 keV), the spectrum of IC 4329A is best described by an absorbed power law with a photon index $\Gamma \simeq 1.83 \pm 0.4$ plus an absorption edge around $0.7 \mathrm{keV}$ having an optical depth of $\simeq 0.6 \pm 0.2$ consistent with absorption by OVI and OVII (Madejski et al. 1995). However, an alternative explanation of the soft excess in terms of a black-body or a steep power law is also allowed by the same data. 
All fits derive a column density of a few $10^{21} \mathrm{~cm}^{-2}$. This column density is largely in excess of the Galactic value $N_{\text {Hgal }} \simeq 4.55 \times 10^{20} \mathrm{~cm}^{-2}$ (Elvis, Lockman \& Wilkes 1989) and indicate absorption intrinsic to IC 4329A. This result is consistent with the fact that its host galaxy is seen edge-on (Petre et al. 1984).

In the following, we present the results from the X-ray observation of IC 4329A with ASCA (Tanaka, Inoue \& Holt 1994). The data between 2 - $10 \mathrm{keV}$ have been previously discussed in a letter by Mushotsky et al. (1995). We now report a more detailed analysis of the overall (0.4-10 keV) data. We show very strong evidence that the X-ray spectrum of IC 4329A is absorbed by highly ionized gas (warm absorber), characterized by the absorption edges of highly ionized oxygen ions (section 3.1). We also confirm the presence of a spectral hardening above $\sim 6-7 \mathrm{keV}$, as well as the iron K-shell emission line, which are characteristics of a reflection spectrum (section 3.2). Complex warm absorber models and accretion-disk emission line models are then discussed (section 4). We conclude that there is evidence of a warm absorber, a strong reflection component and a marginally broad iron fluoresence line in the X-ray spectrum of IC 4329A.

\section{The X-ray data}

The X-ray observation of IC 4329A reported here was carried out with ASCA on August 15th 1993 during the performance verification (PV) phase. Solid state imaging spectrometer (SIS) data were collected in 4-CCD 'Bright' and 'Faint' modes. Faint data were converted into Bright data. No echo corrections nor Dark Frame Error (DFE) corrections were applied (see Otani \& Dotani 1994 for details on these effects). Standard selection criteria were used, the most relevant being elevation and bright Earth angles greater than $5^{\circ}$ and $25^{\circ}$, respectively, and a magnetic cut-off rigidity greater than 8 GeV/c. These were applied to both GIS and SIS data. The resulting effective exposure times are $\sim 29000 \mathrm{~s}$ for the SIS and $\sim 32500 \mathrm{~s}$ for the GIS. About 55000 and 90000 source plus background counts were collected from each SIS and GIS, respectively. The background count rates were about $1 \%$ of the SIS average source count rate $(\sim 1.7$ counts/s) and about $5 \%$ of the GIS average source count rate $(\sim 2.6$ counts $/ \mathrm{s})$. 
Only chip n.1 of SIS0 and chip n.3 of SIS1 were used in the SIS analysis. This results in the loss of $\sim 30 \%$ of the total SIS source counts to the neighboring chips due to the broad wings of the mirror point response function. Source counts were extracted from circular regions of radius $\sim 12$ arcmin and $\sim 6$ arcmin for the gas imaging spectrometer (GIS) and SIS, respectively. Because the source is bright and fills the chip, SIS background was extracted from the SIS blank sky files from a same area as that used for the source. GIS background counts were accumulated from annular regions around the source, with an internal radius of $\sim 14$ arcmin and an external radius of $\sim$ 17 arcmin. Results with blank sky GIS backgrounds are consistent with the reported one to within $\sim 5 \%$. It has been reported from ROSAT observations (Madejski et al. 1995) that two other X-ray sources (the Elliptical Galaxy IC4329 and an unidentified source) lie within $\sim 13$ arcmin of IC 4329A. In the following analysis, we will assume that their contribution is negligible since they become important only below $\sim 0.5 \mathrm{keV}$, as pointed out by Madejski et al. (1995). Neither source is spatially resolved with the SIS nor with the GIS, even when we considered only the soft energies $(\mathrm{E} \lesssim 0.5 \mathrm{keV})$ or only the hard energies $(\mathrm{E} \gtrsim 5 \mathrm{keV})$.

Although the source flux decreased by $\sim 15 \%$ in a timescale of $\sim 1$ day in all detectors, a hardness ratio analysis reveals no significant spectral variation during the observation. Therefore, all photons have been accumulated together for the spectral analysis.

\section{Spectral analysis}

GIS2/3 and SIS0/1 pulse height spectra were binned in order to have a number of counts/bin $\gtrsim 200$ and $\gtrsim 100$ in the energy ranges $(0.7-10 \mathrm{keV})$ and $(0.4-10 \mathrm{keV})$, respectively. Data preparation and spectral analysis have been performed using version $1.0 \mathrm{~h}$ of the XSELECT package and version 8.5 of the XSPEC program (Arnaud et al. 1991). We made use of the response matrices gisv3_1.rmf (released in March 1994) for the GIS detectors and rsp1.1alpha (released in June 1994) with Dark Frame Error (DFE) shifted by $-2 \mathrm{ADU}(\sim 7 \mathrm{eV})$ and $-5 \mathrm{ADU}(\sim 18 \mathrm{eV})$ for the SIS0 and SIS1 detectors, respectively. 
For the GIS data, we first modelled the overall $(0.7$ - $10 \mathrm{keV})$ spectrum with a single absorbed power law plus iron emission line. The column density, $N_{\mathrm{H}}$, was free to vary. This model gives an acceptable $\left(\chi_{\text {red }}^{2} /\right.$ d.o.f. $\left.\simeq 1.33 / 564\right)$ description of the spectrum with $\Gamma \simeq 1.79 \pm 0.02$, a column density $N_{\mathrm{H}} \simeq(3.8 \pm 0.2) \times 10^{21} \mathrm{~cm}^{-2}$ and a very broad $(\sigma \simeq 0.53 \pm 0.24 \mathrm{keV})$ iron emission line at $\mathrm{E} \simeq 6.19 \pm 0.15 \mathrm{keV}$ with $\mathrm{EW} \simeq 233 \pm 80 \mathrm{eV}$ (see Table 1A). With these values, the unabsorbed flux in the 2 - $10 \mathrm{keV}$ energy band is $\simeq 1.01 \times 10^{-10} \mathrm{erg} \mathrm{cm}^{-2} \mathrm{~s}^{-1}$, which corresponds to a luminosity $\mathrm{L}_{2-10 \mathrm{keV}} \sim 1.07 \times$ $10^{44} \mathrm{erg} \mathrm{s}^{-1}$. Although the continuum shape is approximately consistent with previous measurements with the same model (PYM90, Madejski et al. 1995), the iron line appears much broader and a factor of $\sim 2$ stronger than previous values (PYM90, Nandra \& Pounds 1994). However, this difference can be easily understood if we consider that, as shown later on, the SIS data strongly requires the addition of a reflection component to the continuum emission (section 3.2). Because of its better resolution, the SIS can indeed better separate the Fe K line from the reflection component. When the reflection is included in the model, the Fe $\mathrm{K}$ line parameters obtained from the GIS data are considerably reduced (Table 1B) and become consistent with Ginga and SIS results. A detailed description of the reflection parameters given in Table 1B, as well as the full justification for the use of this model, is presented in section 3.2 , in the light of the results obtained from the SIS spectrum.

For the SIS data, a single absorbed power law model with $N_{\mathrm{H}} \sim 4 \times 10^{21} \mathrm{~cm}^{-2}$ and $\Gamma \sim 1.75$ gives an unacceptable fit, with $\chi_{\text {red }}^{2} /$ d.o.f. $\simeq 2.21 / 551$. The residuals to this fit between 0.4 - $10 \mathrm{keV}$ are shown in Figure 1 and are indicative of the complexity of the X-ray spectrum of IC 4329A. At soft energies, the residuals show the presence of excess emission at $\mathrm{E} \lesssim 0.8 \mathrm{keV}$ and absorption features around $1 \mathrm{keV}$. These indicate either that the underlying soft continuum emission is more complex than a single power law or that the absorption is more complex than a uniform, cold absorption. At higher energies, we find an $\mathrm{Fe} \mathrm{K}$ emission line at $\mathrm{E} \sim 6.2 \mathrm{keV}$ but also systematic positive residuals starting from $\mathrm{E} \gtrsim 5 \mathrm{keV}$. The presence of this "hard tail" will appear more evident (section 3.1.2.) after fitting adequately the soft energy part of the spectrum.

Because of the complexity of the SIS spectrum, we split the following analysis in two parts: (i) fit of the spectrum between $0.4-5 \mathrm{keV}$ in order to focus only into the 
characteristics of the soft energy band, (ii) fit of the overall (0.4-10 keV) spectrum.

\subsection{Soft energy band}

\subsubsection{Thermal component}

One possibility to explain the measured soft excess would be to assume a two component continuum emission instead of a single power law. A steep, ultra soft excess may be associated with emission from the inner parts of an accretion disk (Wilkes \& Elvis 1987, Turner and Pounds 1989). As a first approximation, this soft emission can be parameterized by a low temperature $\left(\mathrm{T} \lesssim 10^{6} \mathrm{~K}, \mathrm{kT} \lesssim 100 \mathrm{eV}\right)$ blackbody. A model consisting of a blackbody plus absorbed power law was fitted to the $0.4-5 \mathrm{keV}$ SIS spectrum (SIS0 and SIS1 are fitted simultaneously). The resulting blackbody temperature of $\sim 9.3 \times 10^{5} \mathrm{~K}(\mathrm{kT} \sim 80 \mathrm{eV})$, photon index of $\sim 1.96$ and column density of $\sim 5.8 \times 10^{21} \mathrm{~cm}^{-2}$ are consistent with previous ROSAT results (Madejski et al. 1995), but the residuals shown in Figure $2 a$ present strong and systematic deviations around $\mathrm{E} \simeq 0.6 \mathrm{keV}$ and absorption features around $0.9 \mathrm{keV}$. These clearly indicate that the blackbody model cannot, alone, explain the measured soft excess. The further addition of an absorption edge at $\mathrm{E} \sim 0.76 \mathrm{keV}$ with $\tau \sim 0.46$ improves the statistics of the fit to $\chi_{\text {red }}^{2} /$ d.o.f. $\simeq 1.22 / 481$. In that case, the normalization of the blackbody is reduced by a factor of $\sim 5$ and some systematic structures are still present in the residuals around $0.9 \mathrm{keV}$. These imply that also this possibility is unlikely.

Alternatively, we modelled the soft excess with a Raymond-Smith plasma model (Raymond \& Smith 1986) absorbed by the Galactic column only. This model has been proposed in the past as a possible explanation for the soft excess observed in some Seyfert 1 galaxies (Turner et al. 1991). The best result is obtained allowing the abundances to be free. This gives a plasma temperature of $\sim 2.1 \times 10^{6} \mathrm{~K}(\mathrm{kT} \sim 183 \mathrm{eV})$ and improves the fit to $\chi_{\text {red }}^{2} /$ d.o.f. $\simeq 1.28 / 479$. However, the best-fit abundances are $\sim 1 / 20$ the solar value. Such a required low abundance is obvious from the absence of any blend of emission lines around 0.8-0.9 keV. Moreover, some systematic features are present in the residuals (Figure $2 b$ ). Thus we also exclude this alternative as a possible description of the soft X-ray spectrum of IC 4329A. 


\subsubsection{Absorption edges}

Another possibility to explain the soft excess is an ionized or "warm" absorber. The ionized absorber model assumes that light elements (O, Ne, Mg, Si, Fe) are photoionized and therefore predicts extra emission due to the reduction of the opacity at soft energies. This model also predicts extra absorption around $1 \mathrm{keV}$, mainly due to highly ionized oxygen (Netzer 1993). As clear from the residuals shown in Figure 1, both these features are detected in the spectrum of IC 4329A. These strongly support, a priori, the ionized absorber model.

As a first approximation of a warm absorber, we added a single absorption edge to the absorbed power law model. Given the good statistics of the present data $(\sim 50000$ counts/SIS) and the ASCA spectral resolution (FWHM $\sim 80 \mathrm{eV}$ at $1 \mathrm{keV}$ ), a single absorption edge is a rather crude approximation of a warm absorber, but it allows a direct comparison with previous measurements. The inclusion of an absorption edge in the absorbed power law model improves the fit to $\chi_{\text {red }}^{2} /$ d.o.f. $\simeq 1.30 / 479$ and gives $\mathrm{E}_{\text {edge }} \simeq 0.76 \pm 0.01 \mathrm{keV}$ and $\tau_{\text {edge }} \simeq 0.84 \pm 0.04$. These values are consistent with the ROSAT results (Madejski et al. 1995). However, inspection of the residuals in Figure $2 c$ clearly suggests further complexity. Indeed, the addition of a second absorption edge improves the statistics to $\chi_{\text {red }}^{2} /$ d.o.f. $\simeq 1.15 / 477$, which is significant at more than $99.9 \%$ level $\left(\Delta \chi^{2} \simeq 76\right)$. As evident from the residuals in Figure $2 d$, the two edges plus absorbed power law model provides the best description of the soft X-ray spectrum. Best-fit parameters are reported in Table 2 and confidence contours for the edge energies versus optical depths are shown in Figure 3. Assuming a conservative value of $\sim 10 \mathrm{eV}(1 \sigma)$ for the SIS systematic uncertainties, the edge at $\mathrm{E} \simeq 0.70 \pm 0.02$ $\mathrm{keV}$ is consistent with absorption by $\mathrm{OVI}(671 \mathrm{eV})$, OVII $(739 \mathrm{eV})$ and NVII $(667 \mathrm{eV})$, where the energies quoted in parenthesis are the edge energies in the rest frame (Lotz 1968). The best agreement is with OVII. The second edge at $\mathrm{E} \simeq 0.84 \pm 0.02 \mathrm{keV}$ is consistent with OVIII $(871 \mathrm{eV})$, only. Inspection of the residuals in Figure 5 shows the presence of a further edge-like feature at $\sim 1.25 \mathrm{keV}$, consistent with NeIX(1196 $\mathrm{eV}), \mathrm{NeX}(1362 \mathrm{eV})$ and Fe L edges of Fe ionization stages around FeXVIII. Inclusion of a third absorption edge into the model is, however, significant only at a $\sim 68 \%$ confidence level. Given its low statistical significance, we do not consider it any further 
in the following. It is interesting to note that both characteristic energies obtained for the OVII and OVIII edges are redshifted by approximately the same amount $(\sim 25$ $\mathrm{eV}$, in the observer frame). We checked that this redshift is not related to the Charge Transfer Inefficiency (CTI) of the SIS (see Otani \& Dotani 1994 for details).

Because IC 4329A is a fairly bright point source, we may expect any systematic problems due to remaining calibration uncertainties to be more visible in IC 4329A than in other, weaker sources. We point out that some features are present between $\sim 1.8-2.5 \mathrm{keV}$. These are likely to be due to calibrations uncertainties in this energy range ( $\mathrm{Si} \mathrm{K}$-edge and $\mathrm{Au} \mathrm{M}$-edge of the detector). In this work, we will assume that these features are not of cosmic origin, and are therefore of no physical interest.

Also, even after applying our best-fit edge model (above), there are still residuals in the shape of an emission line around $0.6 \mathrm{keV}$ and an absorption feature around 0.5 $\mathrm{keV}$. In particular, the emission line can be fitted with a narrow $(\sigma \equiv 0)$ gaussian line at $\mathrm{E} \simeq 0.58 \pm 0.05 \mathrm{keV}$ with $\mathrm{EW} \simeq 30 \pm 25 \mathrm{eV}$. The inclusion of this emission line corresponds to a $\Delta \chi^{2} \sim 4$, which is significant at more than 95\%. Its energy is consistent with emission from OVII and/or OVIII. The existence of similar emission lines is predicted in warm absorber models (Netzer 1993) and has been confirmed in the Seyfert 1 galaxy NGC3783 (George, Turner \& Netzer 1995). However, it is also known from other ASCA observations that features around $0.5-0.6 \mathrm{keV}$ are very sensitive to calibration uncertainties (due to the $\mathrm{O}$ K-edge at $\sim 0.54 \mathrm{keV}$ ), background substraction and selection criteria (Miura et al. 1995). Therefore, we have carefully checked to establish the believability of this soft emission line.

When different background regions are used and different selection criteria (like BR_EARTH > 50 for ex.) are applied, both the absorption and soft emission line features remain unchanged. Using the "gain" command in XSPEC, we (linearly) shifted the energies of SIS0 and SIS1 response matrices by $\pm 2 \mathrm{ADU}(\sim 7 \mathrm{eV})$, i.e. within typical systematic errors of $\sim 10 \mathrm{eV}(1 \sigma)$ for the DFE "zero-level” (Otani \& Dotani 1994). After fitting the data again with the shifted matrices, the absorption feature around $0.5 \mathrm{keV}$ appears smoothed out. It is therefore probably due to calibration uncertainties. While the soft emission line changes in intensity it remains statistically significant in every case. However, the emission line is significant only in the SIS1 data. Also, it appears to 
go away when only the night data are considered although, in this case, the statistics are considerably reduced. We conclude that with the present data, the existence of this emission line is doubtful.

\subsection{Overall spectrum}

We now include also the data from $\mathrm{E}>5 \mathrm{keV}$ and proceed to the analysis of the spectrum in the broad $(0.4-10 \mathrm{keV})$ energy band. One striking result is that we detect a clear hardening of the spectrum even down to $5-6 \mathrm{keV}$, likely due to the presence of a reflection component, as previously found by Mushotsky et al. (1995). Figure 4 illustrates the existence of the hard tail and of a Fe $\mathrm{K}$ emission line around $6.2 \mathrm{keV}$. To produce this Figure, the spectrum was fitted between $0.4-5 \mathrm{keV}$ with the two edges plus power law model (section 3.1.2., Table 2) and the data above $5 \mathrm{keV}$ were added back in. The data were rebinned to more than 500 counts/bin in order to better show the presence of the hard tail. The systematic positive values of the residuals, before and after the FeK emission line, are a clear indication that the continuum is increasing at higher energies. This is also confirmed by the huge $(\sigma \sim 1.17 \mathrm{keV}, \mathrm{EW} \sim 760 \mathrm{eV})$ values obtained for the Fe $\mathrm{K}$ line $(\mathrm{E} \sim 6.25 \mathrm{keV})$ if we include only a single gaussian line in the model, which tries to account for the line plus the hard tail. We underline that in this case, as well as in all the following spectral fits, all the parameters of the two edges plus power law in the model are free. Given the importance of the residuals above $7 \mathrm{keV}$, we carried a number of tests to confirm the reality of the hard tail. The use of a background choosed from the outer region of the relevant chip in the SIS had no significant effect on either the overall spectrum or the strenght of the hard tail. The use of different extraction radii for the source (from $\mathrm{r}=4$ arcmin to $\mathrm{r}=9$ arcmin) and more conservative selection criteria (like BR_EARTH>50 to avoid light leakage) gave similar results as well.

In a recent ASCA long observation of the Seyfert 1 galaxy MCG-6-30-15, a very broad iron line has been observed (Tanaka et al. 1995). It has been well modelled by the sum of a broad $(\mathrm{E} \sim 5.6 \mathrm{keV}, \sigma \sim 0.54 \mathrm{keV}, \mathrm{EW} \sim 150 \mathrm{eV})$ and narrow $(\mathrm{E} \sim 6.5$ $\mathrm{keV}, \sigma \sim 0.20 \mathrm{keV}, \mathrm{EW} \sim 150 \mathrm{eV}$ ) gaussian lines. These have been interpreted as the signature of the "double-horned" line expected from an accretion disk (e.g., Fabian et 
al. 1989). If a double-gaussian parameterization is used for IC 4329A instead of a single line, one gaussian converges to a very broad $(\sigma \sim 1.2 \mathrm{keV}, \mathrm{EW} \sim 713 \mathrm{eV})$ line centered at $\sim 6.2 \mathrm{keV}$ and the other one to a narrow $(\sigma=0, \mathrm{EW} \sim 53 \mathrm{eV})$ at the same energy. Since the data require an unusually broad gaussian that is non-physical and inconsistent with any previous X-ray measurement, we prefer to interpret this result as meaning that the high energy part of the spectrum requires an additional continuum component.

The presence of a Fe K emission line and a hard tail is usually interpreted in terms of a model in which the primary continuum emission is reprocessed by optically thick matter, i.e. a reflection component (Lightman \& White 1988, Nandra \& Pounds 1994). Therefore, we added a reflection component in the two edges plus power law model. A gaussian emission line is also included in the fit. The reflection used is the "plrefl" model in XSPEC and is calculated following Lightman \& White (1988). The reflecting matter is assumed to be thick, cold and with cosmic abundances (Morrison and Mc Cammon 1983). The inclination angle $i$ has been fixed to 0, i.e. the reflecting plane is seen face-on, and the covering factor $\Omega / 2 \pi$ has been fixed to 1 . The source is assumed to emit isotropically and the spectrum is integrated over all viewing angles. The relative normalization $\mathrm{R}$ ( $\left.\equiv \mathrm{A}_{\mathrm{refl}} / \mathrm{A}_{\mathrm{pl}}\right)$ between the reflected component $\left(\mathrm{A}_{\text {refl }}\right)$ and the direct component $\left(\mathrm{A}_{p l}\right)$ is a free parameter. This implies that the normalization of the reflection component is allowed to be independent of the normalization of the power law. The parameter $\mathrm{R}$, alone, includes the uncertainties concerning the geometry of the reflector (i.e., the covering fraction), the possibility that the source is anisotropic (e.g., Ghisellini et al. 1991) and time-lag effects. This implies, for example, that a best-fit value of $R$ $\sim 1$ can be explained if the reprocessing material covers $\sim 2 \pi$ of the source. If $\mathrm{R}>$ 2 , then the result cannot be explained only in terms of a covering factor increase, and either the source must be anisotropic or there are time delay effects.

The photon index has been left free to vary. The best-fitting parameters obtained with a reflection fit are given in Table 3. The unfolded spectrum and residuals are shown in Figure 5. The measured photon index of $1.98 \pm 0.05, N_{\mathrm{H}} \simeq(3.48 \pm 0.12) \times 10^{21}$ $\mathrm{cm}^{-2}$ and $\mathrm{Fe} \mathrm{K}$ line parameters $(\mathrm{E} \simeq 6.20 \pm 0.07 \mathrm{keV}, \sigma \simeq 0.11 \pm 0.08 \mathrm{keV}$ and $\mathrm{EW}$ $\simeq 89 \pm 33 \mathrm{eV}$ ) are consistent with previous Ginga results (PYM90, Fiore et al. 1992). Confidence contours in the Fe K parameter space $\sigma$ - E are well constrained (Figure 6). 
These show that the Fe $\mathrm{K}$ line is consistent with redshifted cold iron at more than $90 \%$ confidence level, and is marginally broad at an $\sim 80 \%$ confidence level. As discussed later on, these characteristics of the iron line suggest that we are dealing with a disk geometry (Fabian et al. 1989, Mushotsky et al. 1995).

As already mentioned in section 3, the GIS spectral analysis is completely consistent with that derived above for the SIS data. In fact, the inclusion of a reflection component, assuming an inclination $i=0$ and covering factor $\Omega / 2 \pi=1$ as for the SIS spectrum, improves the GIS fit at a confidence level $>99 \%\left(\Delta \chi^{2} \sim 21\right)$. Comparison of the GIS best-fit parameters (Table 1B) with those obtained from the SIS data (Table 3), shows very good agreement from both detectors and allow us to be fairly confident in the proposed best-fitting model. The major discrepancies are in the $N_{\mathrm{H}}$ and photon index best-fit values which are respectively higher and flatter in the GIS than in the SIS spectrum. However, these differences are likely attributed to the fact that the GIS is not sensitive enough at soft energies to detect the absorption edges. As they are not fitted explicitely in the GIS spectrum, they likely enhance the absorption value and therefore contribute to flatten the GIS spectrum. This hypothesis is also supported by the fact that the fit of the spectrum with all 4 detectors (SIS + GIS) gives results almost identical to that found with the SIS only.

\subsection{On the model-dependence of photon index, reflection nor- malization and $\mathrm{Fe} \mathrm{K}$ line parameters}

With the above best statistical fit of IC 4329A (Table 3), we found that there is a strong (linear) correlation between the photon index and the amount of reflection (Figure 7). This relation should be borne in mind when interpreting the results of this analysis. A similar effect has been discussed for the analysis of MCG-2-58-22 (Weaver et al. 1995). While a high best-fit value of $\mathrm{R}$ may indeed indicate that the true intrinsic photon index is steep, it also depends strongly on fitting the correct amount of reflection, as well as the shape of the reflected spectrum modelled. There is no guarantee that the current

reflection model is entirely correct and we may biasing the fits with a slightly incorrect shape or normalization of the reflection. Different analysis procedures may, therefore, lead to slightly different results. For example, fixing the photon index to its $90 \%$ upper 
limit $(\Gamma \simeq 1.92$ ) obtained from the fit in the $0.4-5 \mathrm{keV}$ energy band (Table 2 ), the amount of reflection converges to $\mathrm{R} \simeq 2.1$. However, fixing the photon index from the fit at soft energies is not necessarily correct in this case because we may also bias our fit with an improper parameterization of the warm absorber. Alternatively, fixing $\mathrm{R}=1$, a value appropriate for a face-on slab subtending $2 \pi$ solid angle at the X-ray source, the fit gives $\Gamma \simeq 1.89$. However, also in this case, there is no reason to fix $\mathrm{R}$. The best solution of the present data is therefore that obtained with $\Gamma$ and $R$ free to vary (Table $3)$.

Because of the correlation between photon index and the amount of reflection in the model we investigated by how much this could affect the parameters obtained for the Fe $\mathrm{K}$ line. For this, we calculated the $90 \%$ confidence contours of $\sigma$ versus energy for Fe K with $\mathrm{R}$ varying from zero (no reflection) to 3.35 (the best-fit value). These contours are shown in Figure 8 for $\mathrm{R}=0,0.3,1,1.5,2.1$ and 3.35. The corresponding best-fit values of the Fe K line width are $1.17,0.89,0.21,0.15,0.13$ and $0.11 \mathrm{keV}$, respectively. The $\chi^{2}$ values are $641,640,639,634,630$ and 626 for 547 d.o.f. We see that the energy of the Fe K line depends only slightly on the exact amount of reflection. These contours are indicative of 3 different "solutions" for the line width: (i) assuming little or no reflection $(\mathrm{R} \lesssim 0.9)$ results in a very broad Fe $\mathrm{K}$ line with characteristic width of $\gtrsim 0.8 \mathrm{keV}$ and $\mathrm{EW} \gtrsim 380 \mathrm{eV}$, (ii) assuming an intermediate contribution from reflection $(\mathrm{R} \sim 1-2)$ results in two local minima, one near $\sigma \sim 0.6-0.8 \mathrm{keV}$ and the other near $\sigma \sim 0.1$ $0.2 \mathrm{keV}$, and (iii) assuming a strong reflection component ( $\mathrm{R}$ from $\sim 2$ to the best-fit value $\sim 3.3)$ results in one minimum at $\sigma \sim 0.11 \mathrm{keV}$ with well constrained contours. Fitting simultaneously the 2 SIS and 2 GIS detectors yielded very similar results. These contours, as well as the best-fit values, are similar to those obtained by Mushotsky et al. (1995) although their fit yields a slighlty larger Fe K width than inferred from our bestfit (case (iii)). The difference may be a consequence of the slightly different continuum modelling, probably caused by the different energy band used by the authors.

To summarize, we find that although the photon index and reflection normalization are strongly coupled together, the most likely description of the spectrum of IC 4329A is given by its best statistical fit (Table 3). We have also shown that despite this intrinsic uncertainty, the Fe K parameters depend only little on the exact amount of reflection, 
provided $\mathrm{R}$ is higher than $\sim 2$, as required by the best-fit model. With these caveats borne in mind, we can discuss and try to interpret these results physically.

\section{Discussion}

Above results show that the best interpretation of the soft X-ray spectrum of IC 4329A is in terms of photoionized absorber. Therefore, we have also fitted the SIS data between 0.4 and $5 \mathrm{keV}$ with a warm absorber model, also including a uniform cold absorber. The model has been produced using the photoionization code CLOUDY (Ferland 1991) and using the assumptions as in Fabian et al. (1994). Free parameters are the ionization parameter $\xi=\mathrm{L} / n R^{2}\left(\mathrm{erg} \mathrm{s}^{-1} \mathrm{~cm}\right)$, the "warm" column density $N_{\mathrm{W}}\left(\mathrm{cm}^{-2}\right)$, and the cold column density $N_{\mathrm{H}}\left(\mathrm{cm}^{-2}\right)$. The photon index has been fixed to 2.0 , a value close to the SIS best-fit value. The fit yields an acceptable fit with $\chi_{\text {red }}^{2} /$ d.o.f. $=1.23 / 480$. Although it is statistically better than with the blackbody model, it is worse than with the two absorption edges. We attribute this difference to the fact that the model used is based on simple basic assumptions which may not reflect the reality, namely: a thin geometry for the photoionized gas, solar abundances, constant temperature, constant density and a source luminosity of $10^{43} \mathrm{erg} \mathrm{s}^{-1}$. Further, the model does not include the emission lines expected from a more realistic warm absorber (Netzer 1993). We obtain $\log \xi \simeq 1.12 \pm 0.04, \log N_{\mathrm{W}} \simeq 21.70 \pm 0.02$ and $\log N_{\mathrm{H}} \simeq 21.49 \pm 0.01$. The fitted neutral column density is again about one order of magnitude higher than the Galactic value.

Interesting is to note that with the 2 edges parameterization of the warm absorber, both edges best-fit energies are redshifted by approximately $25 \mathrm{eV}$. If real, this redshift correspond to a Doppler velocity of about $10000 \mathrm{~km} \mathrm{~s}^{-1}$. Although this result depends strongly on the correct modelling of the warm absorber (e.g., the redshift of the lower edge may be interpreted as a blend of OVI and OVII edges) and on the precise calibration of the energy scale, it may be the signature of infalling matter or largescale turbulance associated to the warm absorber region. Firm conclusions on this point require more investigations, but we point out that edge energies shifted compare to their expected energy have been reported also for the observations of MCG-6-30-15 and NGC4051 
(Otani 1995).

As suggested by Krolik \& Kriss (1995), resonance line scattering may significantly contribute to the total opacity of the warm gas. It is worth noting therefore that it is a potential confusing factor when interpreting the energy and optical depth of the absorption edges. For example, the presence of blended resonance oxygen lines may cause a misidentification of OVIII as OVII due to the apparent energy shift in the onset of the OVIII edge. Alternatively, it could contribute to shift the apparent energies of the absorption edges. Unfortunately, quantitative considerations on resonance absorption being strongly model-dependent (e.g. on the covering factor and velocity of the warm gas), and given the limited spectral resolution of the SIS, we cannot exclude the presence of resonance absorption lines in the present data.

A striking alternative to the warm (plus cold) absorber is absorption by cold matter with abundances free to vary. IC 4329A is known to exhibit absorption of a few $10^{21}$ $\mathrm{cm}^{-2}$. This absorption is consistent with the fact that its host galaxy is seen edge-on, and so we examine whether the absorption edges seen in the ASCA data can be due to neutral interstellar matter. Since the K-edge energies of OVII, OVIII and NeX are close to the L-edge energy of FeI, K-edge energies of NeI and MgI, respectively, it is possible that abundances larger than the cosmic value can mimic the absorption edges normally interpreted as the presence of a warm absorber. In fact, a fit of the soft energy band $(0.4-5 \mathrm{keV})$ with a cold absorber with variable abundances gives an equally good fit as that obtained with our previous parameterization using two warm edges (section 3.1.2). However, extremely high abundances ( $\sim 5-15$ times the cosmic value) of all metals are required. Also, known calibration problems around $0.5-0.6 \mathrm{keV}$ (see section 3.1.2) make the results from this fit doubtful. A deeper investigation using these and other data is currently being conducted and will be presented in a future paper (Hayashida et al., in preparation).

The X-ray spectrum of IC 4329A also requires a remarkably strong reflection component. In a recent re-analysis of $H E A O-1(2-40 \mathrm{keV})$ data, a similar amount (R $\sim 2.3 \div 4.2$ ) of reflection component has been detected in IC 4329A (Weaver, Arnaud, \& Mushotzky 1995). Similarly, we find in the ASCA spectra $\sim 2$ - 3 times more reflection relative to the continuum than in the Ginga observation (PYM90, Fiore et al. 
1992, Nandra \& Pounds 1994). The present data also suggest a slight, but significant, increase in the absolute amount of reflection. Although we might recall that the ASCA best-fit spectra, as well as the $H E A O-1$ and Ginga spectra, exhibit a strong coupling between photon index and amount of reflection (see section 3.3), these results indicate the presence of a strong and variable reflection component. It should be noted that a time-resolved analysis of the Ginga observation also suggested day to day variations in the amount of reflection component in IC 4329A (Fiore et al. 1992).

If the geometry of AGN consists on a source illuminating hard X-rays above and below a soft X-ray emitting disk (e.g. Haardt, Maraschi \& Ghisellini 1994), and these hard X-rays are produced by Inverse Compton scattering of the soft X-rays, then the radiation field within the hard X-ray emitting region is likely to be strongly anisotropic (Ghisellini et al. 1991). The reflecting matter may therefore see more radiation than that emitted towards the observer, by up to a factor of $\sim 5$, as a direct consequence of the electrons losing more energy on head-on collisions with the soft X-ray photons than on collisions from behind. In that case, a strong reflection component becomes physically plausible. However, anisotropy alone is unlikely to explain the increased contribution of the reflected component compared to that of the direct continuum because it requires a change in the anisotropic factor. In other words, anisotropy can explain a high value of $\mathrm{R}$ but not a variation of $\mathrm{R}$. Instead, a time lag effect could, alone, explain all of the observational constraints. Though IC 4329A has never exhibited strong, rapid variability, it has varied up to a factor of $\sim 2.8$ (from a $2-10 \mathrm{keV}$ flux of $\sim 5 \times 10^{-11} \mathrm{erg}$

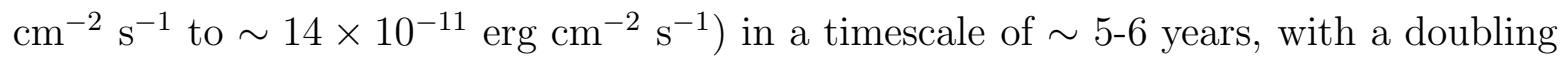
timescale down to $\sim 1$ year, in its $\sim 20$ years history of X-ray observations. A time lag could therefore cause strong amount of reflection. Our results can indeed be understood in this context if the continuum flux decreased by some amount prior to the ASCA observation. Specifically, the flux would have to have decreased by a factor of $\sim 2$ from a time when it was higher than that measured by Ginga, and the reflection component could not have had time to respond to this decrease. This can simultaneously explain a high value of $\mathrm{R}$, a variation of $\mathrm{R}$, and a higher absolute amount of reflection as compared to Ginga.

However, another difficulty with the above results is that strong reflection should be 
accompanied, in most cases, by a strong iron line. We note that the reflected spectrum used in our fitting procedure (section 3.2) is averaged over all viewing angles and has accordingly a slightly different shape than a face-on reflected spectrum. The best-fit amount of reflection $\mathrm{R} \sim 3.3$ corresponds therefore to a $(\sim 1.33$ times $)$ lower value $\mathrm{R} \sim$ 2.5 for a face-on view (Ghisellini, Haardt \& Matt 1994, Magdziarz \& Zdziarski 1995). As a rough estimate, assuming an Fe $\mathrm{K}$ line $\mathrm{EW}$ of $\sim 140 \mathrm{eV}$ in the case $\mathrm{R}=1$ for a cold face-on disk and an incident power law spectrum with $\Gamma \sim 2.0$, we expect an EW of $\sim 300 \mathrm{eV}$. The best-fit amount of reflection requires therefore $\sim 3$ times more line than observed $(\mathrm{EW} \simeq 89 \pm 33 \mathrm{eV})$. This difference may be reconciled by reducing the abundance of iron, or by increasing the abundance of the light metals (lighter than iron) (Reynolds, Fabian \& Inoue 1995). Alternatively, if the Fe K line arises in an ionized disk, its EW can be very low even in the presence of strong reflection because of either resonant trapping of line photons or complete ionization of iron (Ross \& Fabian 1993, Życki \& Czerny 1994). Compare to the neutral case, the ionized disk has lower opacity at lower energies. This might help to explain why the reflection shows up at such low $(\sim 5-6 \mathrm{keV})$ energies in this source. However, we show below that the above constraints can be considerably reduced because the EW obtained with an accretion disk line model is significantly higher.

The parameters of the Fe K line obtained from the SIS spectral analysis are consistent with the line being redshifted by $\sim 100 \mathrm{eV}$ and slightly broad $(\sigma \sim 110 \mathrm{eV})$. These support the idea that a fit of the line with a disk line model (Fabian et al. 1989) would be more appropriate than a gaussian fit. A disk line model takes into account the effects of doppler-broadening and gravitational and transverse redshifts in order to calculate the characteristic line profile. The resulting line profile is highly asymmetric, and often "double-horned". A remarkable example of the applicability of this model to Seyfert 1 galaxies has come from ASCA long observations of MCG-6-30-15 (Tanaka et al. 1995).

We attempted a fit of the SIS data with the "diskline" model available in the XSPEC program. This model calculates the intensity and profile of the line from a flat, optically thick accretion disk as calculated in Fabian et al. (1989). The details of the line depend on the inclination $i$ of the disk, the inner $\mathrm{R}_{\text {in }}$ and outer $\mathrm{R}_{\text {out }}$ disk radii, and on the line emissivity $\alpha$ (assumed to vary as $\mathrm{R}^{-\alpha}$ ). Following Mushotsky et al. (1995), we fix 
$R_{\text {in }}=3 R_{s}$ and $R_{\text {out }}=1000 R_{s}$, where $R_{s}$ is the Schwarzschild radius of the black hole $\left(\mathrm{R}_{\mathrm{s}} \sim 3 \times 10^{5} \mathrm{M} / \mathrm{M}_{\odot} \mathrm{cm}\right.$, with $\mathrm{M}$ the mass of the black hole). The inclination of the disk, $i$, is also fixed at 0 (face-on) to be consistent with our assumption of a face-on reflection component. The line energy is fixed to 6.3 which correspond to the redshifted energy of a fluorescence Fe K line. Only the normalization of the line and its emissivity $\alpha$ are free to vary. The fit yields $\alpha \simeq 2.30 \pm 0.20$ and an EW of $188 \pm 60 \mathrm{eV}$. If $i$ is allowed to vary, we obtain an upper limit of $i \lesssim 25$ degrees which confirms a posteriori that the assumption of a disk face-on is substantially correct. In this case, the EW is enhanced to $213_{-63}^{+76} \mathrm{eV}$. These results are characteristic of a disk observed face-on, in which most of the fluorescence Fe $\mathrm{K}$ line is emitted within a few tens of Schwarzschild radii. As stated above, with the use of a disk line model, the Fe K EW is in rough agreement with the value expected from the reflection component.

Strictly speaking, if a disk line model is included, we should also take into account relativistic effects on the (continuum) reflection component in order to have a selfconsistent model. This model is not available at the moment. This problem will be explored in a later paper. However, relativistic corrections should produce merely an energy shift and/or broadening of the reflection component. Therefore, we expect these shouldn't significantly affect the above results.

It should be noted however that the velocity width $\left(\mathrm{FWHM} \simeq 12000 \pm 8500 \mathrm{~km} \mathrm{~s}^{-1}\right)$ of the Fe $\mathrm{K}$ line is still consistent with an average derived FWHM of $\simeq 4070 \pm 30 \mathrm{~km}$ $\mathrm{s}^{-1}$ for the strong Balmer lines $\left(\mathrm{H}_{\alpha}, \mathrm{H}_{\beta}\right.$ and $\left.\mathrm{H}_{\gamma}\right)$ which dominate the optical spectrum of IC 4329A. This suggests that the Fe K line might, alternatively, be produced from the broad line region. One-sided beaming of the continuum emission and/or obscuration may be necessary, however, to explain the apparent redshift of the line.

\section{Conclusions}

To summarise, the present observation has revealed the following.

(i) The apparent soft X-ray spectrum of IC 4329A is complex with a predominant soft-excess (compared to a simple absorbed power law) at $\mathrm{E} \lesssim 0.8 \mathrm{keV}$ and deep absorption structures between $\sim 0.8-1.2 \mathrm{keV}$. The unprecedent high $(>10$ in every channel) 
signal to noise ratio of this observation allows us to discard, directly from the residuals, a blackbody emission model as an alternative explanation for the soft excess. The soft X-ray spectrum is instead best described by two absorption edges at $\mathrm{E} \sim 0.70 \mathrm{keV}$ and $\mathrm{E}$ $\sim 0.84 \mathrm{keV}$, consistent with absorption by OVI, OVII or NVII, and OVIII, respectively. This complex absorption is clearly the signature of a warm absorber, confirming and extending previous results obtained from ROSAT observations (Madejski et al. 1995).

(ii) Confirmation of the existence of a "hard tail" at higher energies, characteristic of a reflection component (Mushotsky et al. 1995). The hard tail is clearly detected in both GIS and SIS, at similar intensities. When the reflection is included, we find that the data prefer a stronger absolute amount of reflection and stronger contribution relative to the direct continuum than previously observed. A time lag effect can account for all observational constraints.

(iii) Because of the presence of the reflection component, the Fe $\mathrm{K}$ line width and equivalent width are model-dependent. These are, however, only weakly modeldependent provided $\mathrm{R}$ is higher than $\sim 2.1$ (section 3.3). With the proposed best-fit model (with $\mathrm{R} \simeq 3.3 \pm 1.1$ ), we find evidence that the Fe $\mathrm{K}$ line is redshifted (by $\sim 100$ $\mathrm{eV}$ ) and slightly broad $(\sigma \simeq 0.11 \pm 0.08 \mathrm{keV})$, as expected if the emission line is emitted by an accretion disk. The weak $(\mathrm{EW} \simeq 89 \pm 33 \mathrm{eV}) \mathrm{Fe} \mathrm{K}$ (gaussian) line requires iron underabundance or/and ionized matter to be consistent with strong reflection. A fit with a diskline model which includes the wings of the line can, however, account for most of the discrepancy as well.

\section{Acknowledgements}

M.C. thanks K. Leighly and S. Molendi for helpful discussions. M.C. also acknowledges financial support from the Science and Technology Agency of Japan (STA fellowship), hospitality from the RIKEN Institute and support from the European Union. The authors also thank an anonymous referee for valuable comments. 


\section{$7 \quad$ References}

Arnaud, K.A., Haberl, F., \& Tennant, A., 1991, XSPEC User's Guide, ESA TM-09

Elvis, M., Lockman, F.J., \& Wilkes, B.J., 1989, AJ, 97, 777

Fabian, A.C., Rees, M.J., Stella, L. \& White, N.E., 1989, MNRAS, 238, 729

Fabian, A.C. et al., 1994, PASJ, 46, L59

Ferland, G.L., 1991, Ohio State Univ., Astronomy Dept. Internal Report 91-01

Fiore, F., Perola, G.C., Matsuoka, M., Yamauchi, M., \& Piro, L., 1992, A\&A, 262, 37

George, I.M., Turner, T.J., \& Netzer, H., 1995, ApJL, 438, L67

George, I.M., \& Fabian, A.C., 1991, MNRAS, 249, 352

Ghisellini, G., George, I.M., Fabian, A.C., \& Done, C., 1991, MNRAS, 248, 14

Ghisellini, G., Haardt, F., \& Matt, G., 1994, MNRAS, 267, 743

Guainazzi, M., Matsuoka, M., Piro, L., Mihara, T., \& Yamauchi, M., 1994, ApJ, 436, L35

Guainazzi, M., et al. 1995, in preparation

Guilbert, P.W., \& Rees, M.J., 1988, MNRAS, 233, 475

Haardt, F., Maraschi, L., \& Ghisellini, G., 1994, ApJL, 432, L95

Hayashida, K., et al., 1995, in preparation

Krolik, J.H., \& Kriss, G.A., 1995, ApJ, 447, 512

Leighly, K., et al., 1995, submitted to ApJ

Lightman, A.P. \& White, T.R., 1988, ApJ, 335, 57

Lotz, W., 1968, J. Opt. Soc. Am., 58, 915

Madejski, G.M., et al. 1995, ApJ, 438, 672

Magdziarz, P., \& Zdziarski, A.A., 1995, MNRAS, in press

Matsuoka, M. 1994, in "New Horizon of X-ray Astronomy”, ed. F. Makino \& T. Ohashi,

(Tokyo: Universal Academy Press), p305

Mihara, T., Matsuoka, M., Mushotsky, R.F., Kunieda, H., Otani, C., Miyamoto, S., \&

Yamauchi, M., 1994, PASJ, 46, L137

Miura, N., et al. 1995, in preparation

Miyoshi, S., et al., 1988, PASJ, 40, 127

Morrison, R., \& McCammon, D., 1983, ApJ, 270, 119 
Mushotsky, R.F., Fabian, A.C., Iwasawa, K., Kunieda, H., Matsuoka, M., Nandra, K., \& Tanaka, Y., 1995, MNRAS, 272, L9

Nandra, K., \& Pounds, K.A., 1994, MNRAS, 268, 405

Netzer, H., 1993, ApJ, 411, 594

Otani, C., 1995, PhD Thesis

Otani, C., \& Dotani, T., 1994, Asca News n.2, 25

Piro, L., Yamauchi, M., \& Matsuoka, M., 1990, ApJ, 360, L35 (PYM90)

Petre, R., Mushotsky, R.F., Krolik, J., \& Holt, S.S., 1984, ApJ, 280, 499

Ptak, A., Yaqoob, T., Serlemitsos, P.J., Mushotsky, R.F., \& Otani, C., 1994, ApJ, 436, L31

Raymond, J.C. \& Smith, B.W., 1977, ApJS, 35, 419

Reynolds, C.S., Fabian, A.C. \& Inoue, H., 1995, MNRAS, in press

Ross, R.R., \& Fabian, A.C., 1993, MNRAS, 261, 74

Tanaka, Y., Inoue, H., \& Holt, S.S., 1994, PASJ, 46, 37

Tanaka, Y. et al., 1995, Nature, 375, 659

Turner, T.J., Weaver, K.A., Mushotsky, R.F., Holt, S.S., \& Madejski, G.M., 1991, ApJ, 381,85

Turner, T.J., \& Pounds, K.A., 1989, MNRAS, 240, 833

Weaver, K.A., Arnaud, K.A., \& Mushotzky, R.F. 1995, ApJ, in press

Weaver, K.A., Nousek, J., Yaqoob, T., Hayashida, K., \& Murakami, S., 1995, ApJ, submitted

Wilkes, B.J., \& Elvis, M., 1987, ApJ, 323, 243

Wilson, A.S., \& Penston, M.V., 1979, ApJ, 232, 389

Życki, P.T., \& Czerny, B., 1994, MNRAS, 266, 653 
TABLE 1 - GIS2+3 Data $(0.7-10 \mathrm{keV})$

A. Results of Power-Law + Fe K Line Fits

\begin{tabular}{ccccccc}
\hline \hline $\begin{array}{c}N_{\mathrm{H}} \\
\left(10^{21} \mathrm{~cm}^{-2}\right)\end{array}$ & $\Gamma$ & $\mathrm{A}_{\mathrm{pl}}^{a}$ & $\begin{array}{c}\mathrm{E}(\mathrm{FeK}) \\
(\mathrm{keV})\end{array}$ & $\begin{array}{c}\sigma(\mathrm{FeK}) \\
(\mathrm{keV})\end{array}$ & $\begin{array}{c}\mathrm{EW}(\mathrm{FeK}) \\
(\mathrm{eV})\end{array}$ & $\chi_{\text {red }}^{2} /$ d.o.f. \\
\hline $3.78_{-0.12}^{+0.18}$ & $1.79_{-0.03}^{+0.02}$ & $27.6_{-0.7}^{+0.7}$ & $6.19_{-0.15}^{+0.12}$ & $0.53_{-0.24}^{+0.24}$ & $233_{-73}^{+95}$ & $1.33 / 564$ \\
\hline \hline
\end{tabular}

B. Results of Power Law + Fe K Line + Reflection Fits

\begin{tabular}{cccccccc}
\hline \hline $\begin{array}{c}N_{\mathrm{H}} \\
\left(10^{21} \mathrm{~cm}^{-2}\right)\end{array}$ & $\Gamma$ & $\mathrm{A}_{\text {refl }}^{a}$ & $\mathrm{R}^{b}$ & $\begin{array}{c}\mathrm{E}(\mathrm{FeK}) \\
(\mathrm{keV})\end{array}$ & $\begin{array}{c}\sigma(\mathrm{FeK}) \\
(\mathrm{keV})\end{array}$ & $\begin{array}{c}\mathrm{EW}(\mathrm{FeK}) \\
(\mathrm{eV})\end{array}$ & $\chi_{\text {red }}^{2} /$ d.o.f. \\
\hline $4.22_{-0.27}^{+0.24}$ & $1.89_{-0.04}^{+0.05}$ & $73_{-28}^{+28}$ & $2.43_{-0.95}^{+1.04}$ & $6.22_{-0.08}^{+0.08}$ & $\lesssim 0.26$ & $78_{-24}^{+38}$ & $1.30 / 563$ \\
\hline \hline
\end{tabular}

Note: Intervals are at $90 \%$ confidence for one interesting parameter.

${ }^{a}$ Normalization in $10^{-3}$ photons $\mathrm{cm}^{-2} \mathrm{~s}^{-1} \mathrm{keV}^{-1}$ at $1 \mathrm{keV}$.

${ }^{b} \mathrm{R} \equiv \mathrm{A}_{\text {refl. }} / \mathrm{A}_{\mathrm{pl}}$ (see section 3.2 for details)

TABLE 2 - SIS0+1 Data $(0.4-5 \mathrm{keV})$

Results of Two Edges + Power Law Fits

\begin{tabular}{cccccccc}
\hline \hline $\begin{array}{c}\mathrm{E}_{1} \\
(\mathrm{keV})\end{array}$ & $\tau_{1}$ & $\begin{array}{c}\mathrm{E}_{2} \\
(\mathrm{keV})\end{array}$ & $\tau_{2}$ & $\begin{array}{c}N_{\mathrm{H}} \\
\left(10^{21} \mathrm{~cm}^{-2}\right)\end{array}$ & $\Gamma$ & $\mathrm{A}_{\mathrm{pl}}^{a}$ & $\chi_{\text {red }}^{2} /$ d.o.f. \\
\hline $0.70_{-0.03}^{+0.01}$ & $0.63_{-0.08}^{+0.09}$ & $0.84_{-0.02}^{+0.01}$ & $0.39_{-0.07}^{+0.06}$ & $3.30_{-0.09}^{+0.08}$ & $1.89_{-0.03}^{+0.03}$ & $29.5_{-0.7}^{+0.7}$ & $1.15 / 477$ \\
\hline \hline
\end{tabular}

Note: Intervals are at $90 \%$ confidence for one interesting parameter.

${ }^{a}$ Normalization in $10^{-3}$ photons $\mathrm{cm}^{-2} \mathrm{~s}^{-1} \mathrm{keV}^{-1}$ at $1 \mathrm{keV}$. 
TABLE 3 - SIS0+1 Data $(0.4-10 \mathrm{keV})$

Results of Two Edges + Power Law + Fe K Line + Reflection Fits

\begin{tabular}{cccccccccc}
\hline \hline $\begin{array}{c}\mathrm{E}_{1} \\
(\mathrm{keV})\end{array}$ & $\tau_{1}$ & $\begin{array}{c}\mathrm{E}_{2} \\
(\mathrm{keV})\end{array}$ & $\tau_{2}$ & $\begin{array}{c}N_{\mathrm{H}} \\
\left(10^{21} \mathrm{~cm}^{-2}\right)\end{array}$ & $\Gamma$ & $\mathrm{A}_{\text {refl }}^{a}$ & $\mathrm{R}^{b}$ & $\begin{array}{c}\mathrm{E}(\mathrm{FeK}) \\
(\mathrm{keV})\end{array}$ & $\begin{array}{r}\sigma(\mathrm{FeK} \\
(\mathrm{keV}\end{array}$ \\
\hline $0.70_{-0.02}^{+0.01}$ & $0.67_{-0.09}^{+0.07}$ & $0.84_{-0.02}^{+0.01}$ & $0.40_{-0.07}^{+0.07}$ & $3.48_{-0.13}^{+0.12}$ & $1.98_{-0.05}^{+0.05}$ & $102_{-34}^{+41}$ & $3.35_{-1.10}^{+1.17}$ & $6.20_{-0.06}^{+0.07}$ & $0.11_{-0}^{+0}$ \\
\hline \hline
\end{tabular}

Note: Intervals are at $90 \%$ confidence for one interesting parameter.

${ }^{a}$ Normalization in $10^{-3}$ photons $\mathrm{cm}^{-2} \mathrm{~s}^{-1} \mathrm{keV}^{-1}$ at $1 \mathrm{keV}$.

${ }^{b} \mathrm{R} \equiv \mathrm{A}_{\mathrm{reff} .} / \mathrm{A}_{\mathrm{pl}}$ (see section 3.2 for details) 


\section{Figure captions}

Figure 1: Residuals of the SIS spectrum between 0.4 - $10 \mathrm{keV}$, for a model consisting of a single absorbed power law with $\Gamma \sim 1.75$. This figure illustrates the soft excess, absorption features around $1 \mathrm{keV}, \mathrm{Fe} \mathrm{K}$ line at $\sim 6.2 \mathrm{keV}$ and hard tail starting at $\mathrm{E} \gtrsim$ $5 \mathrm{keV}$ (see also Fig. 4).

Figure 2: Residuals of the SIS spectrum between 0.4 - $5 \mathrm{keV}$ for a model consisting

of: (a) absorbed blackbody plus power law, (b) absorbed power law plus Raymond Smith spectrum covered by only the Galactic column density, (c) absorption edge plus absorbed power law and $(d)$ two absorption edges plus absorbed power law.

Figure 3: Contour plots illustrating the (observed) energy and optical depth 68, 90 and 99 per cent confidence levels of the two absorption edges at $(a) \mathrm{E} \sim 0.7 \mathrm{keV}$ and (b) $\mathrm{E} \sim 0.84 \mathrm{keV}$. The redshifted energies of the absorption edges have been marked in the figures.

Figure 4: Residuals of the SIS spectrum between 0.4 - $10 \mathrm{keV}$, for a model consisting of a two absorption edges plus an absorbed power law fitted between $0.4-5 \mathrm{keV}$. The data have been rebinned with more than 500 counts/bin to better show the systematic increase of the residuals above $5 \mathrm{keV}$.

Figure 5: SIS0+1 unfolded spectrum, best-fitting power law plus two absorption edges plus Fe K line plus reflection model (upper panel). Residuals in the form of the ratio (data/model) are shown in the lower panel.

Figure 6: Fe K width and energy 68, 90 and 99 per cent confidence contour levels for the best fit model, as given in Table 3 .

Figure 7: Contours at 68, 90 and 99 per cent confidence of amount of reflection (R) vs. photon index $(\Gamma)$. They illustrate the strong correlation between the two parameters.

Figure 8: Contours at 90 per cent confidence of amount of line width, $\sigma$, vs. observed line energy calculated for various amounts of reflection. The best-fit values are marked with crosses. 


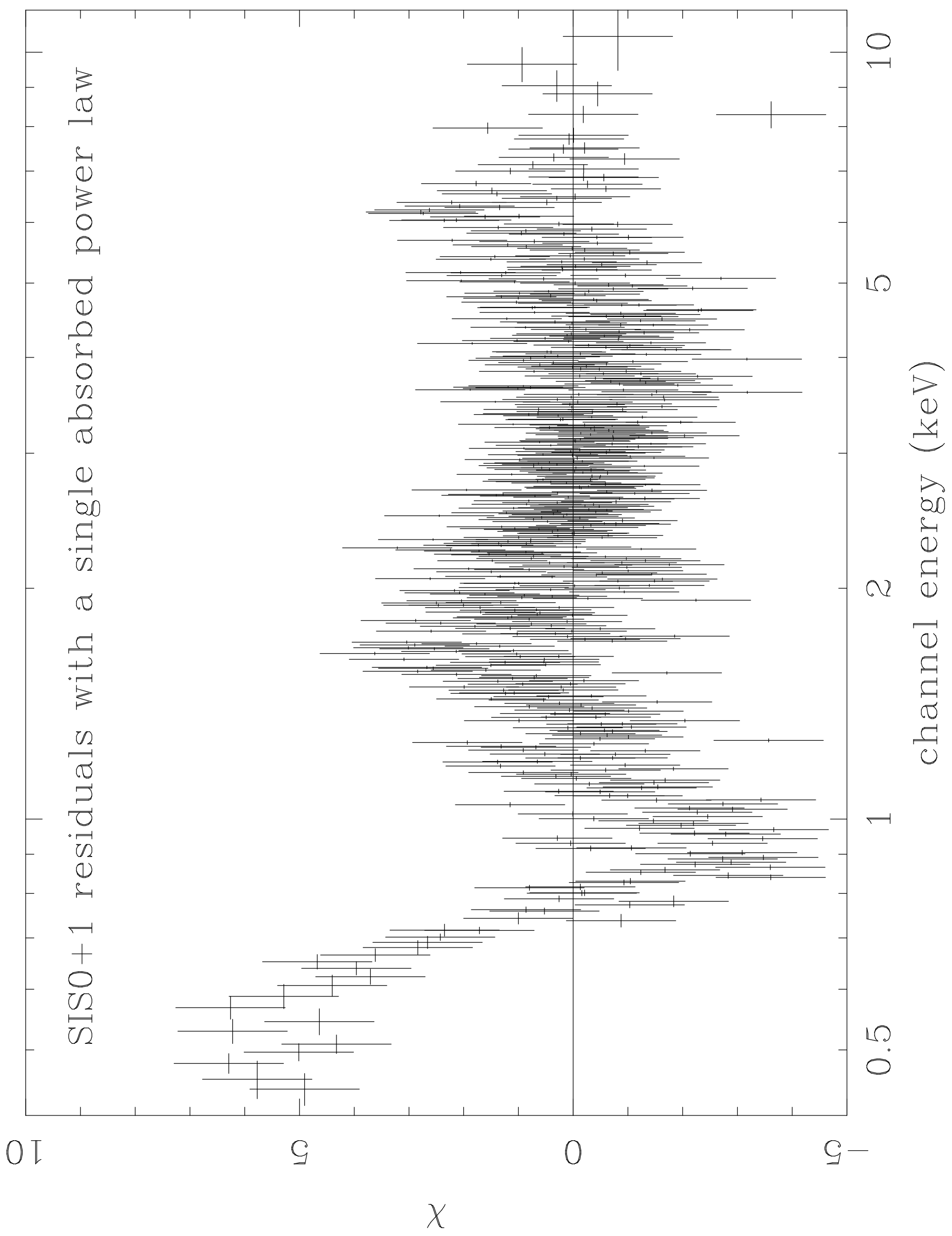




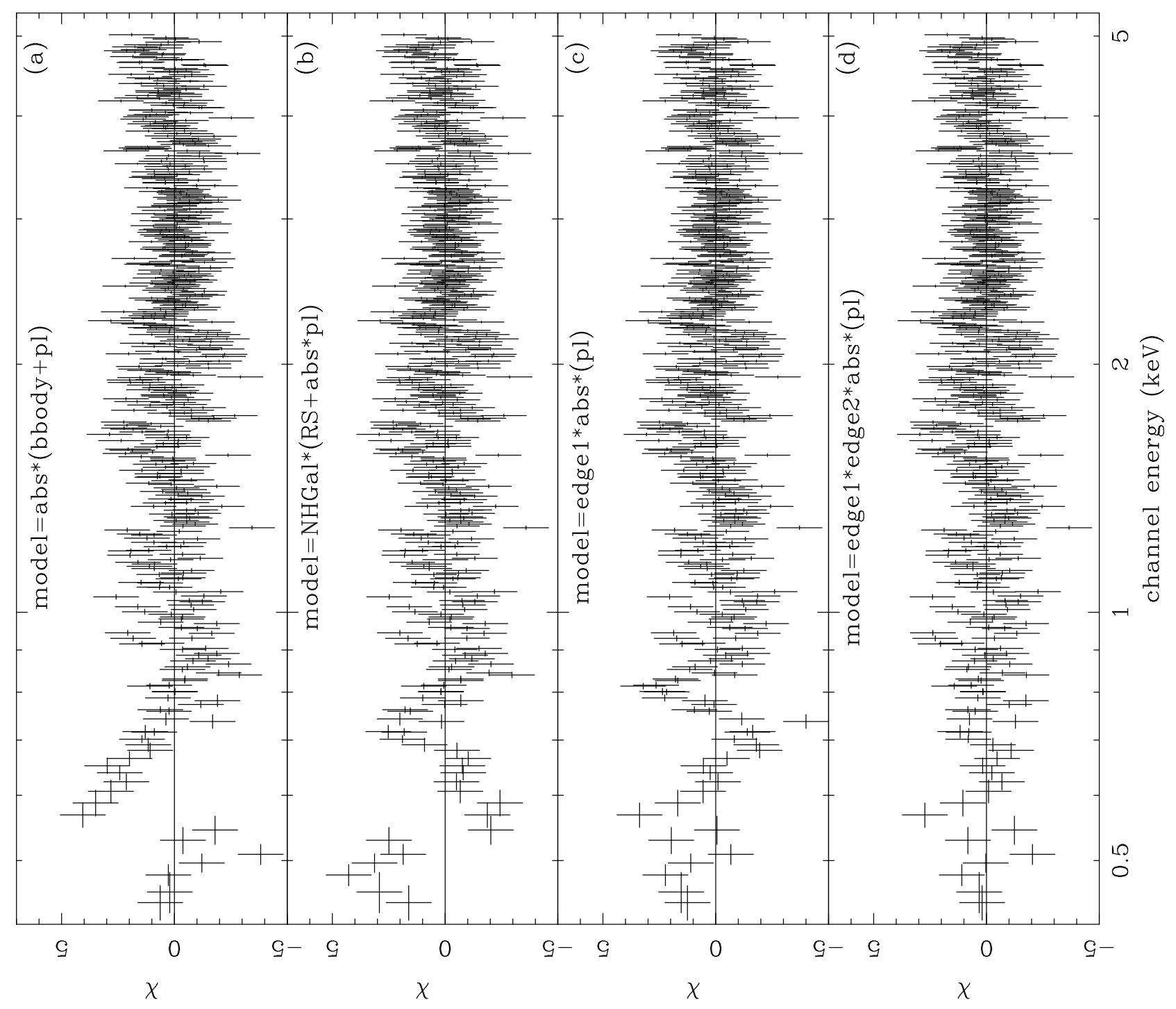




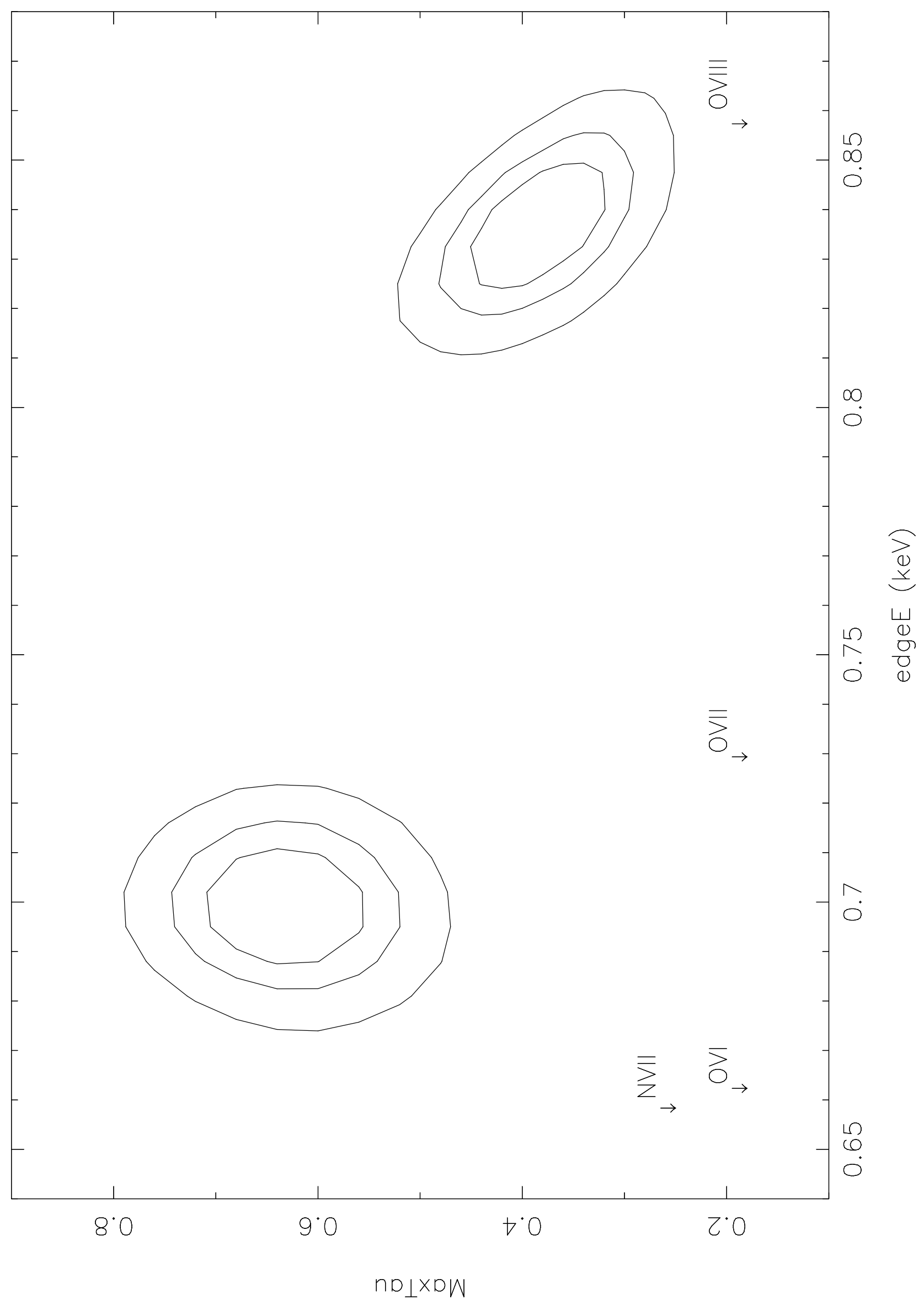




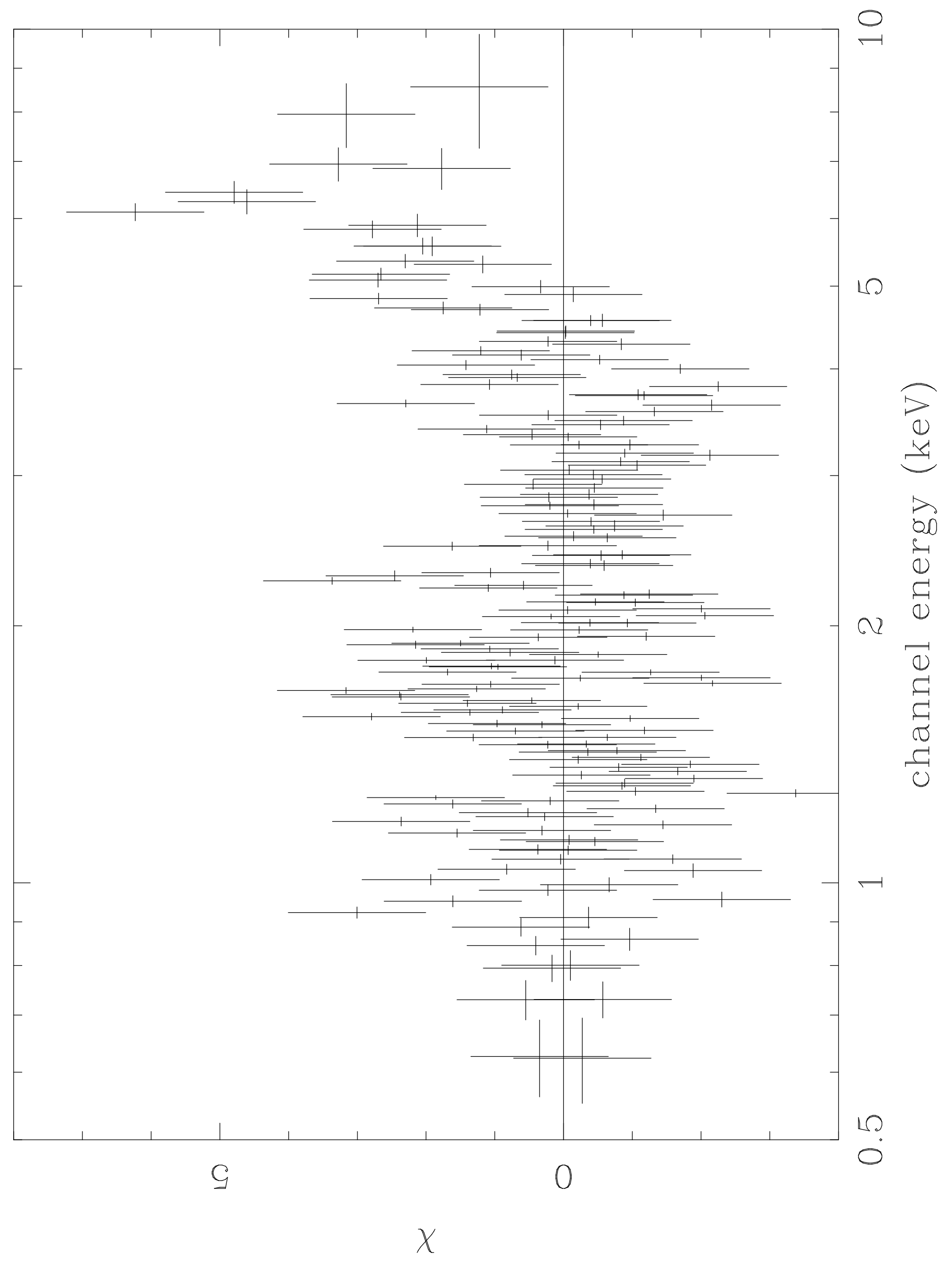




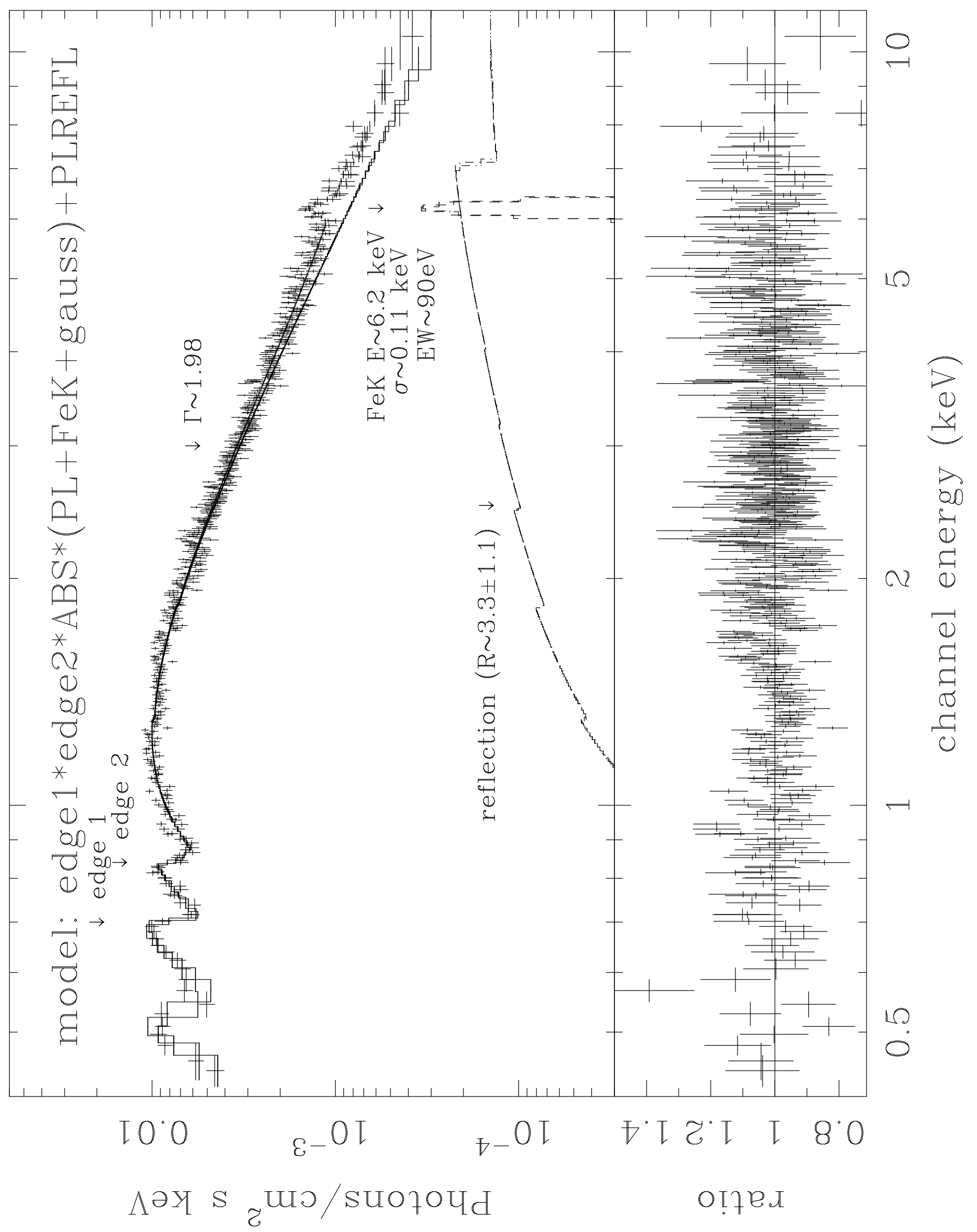




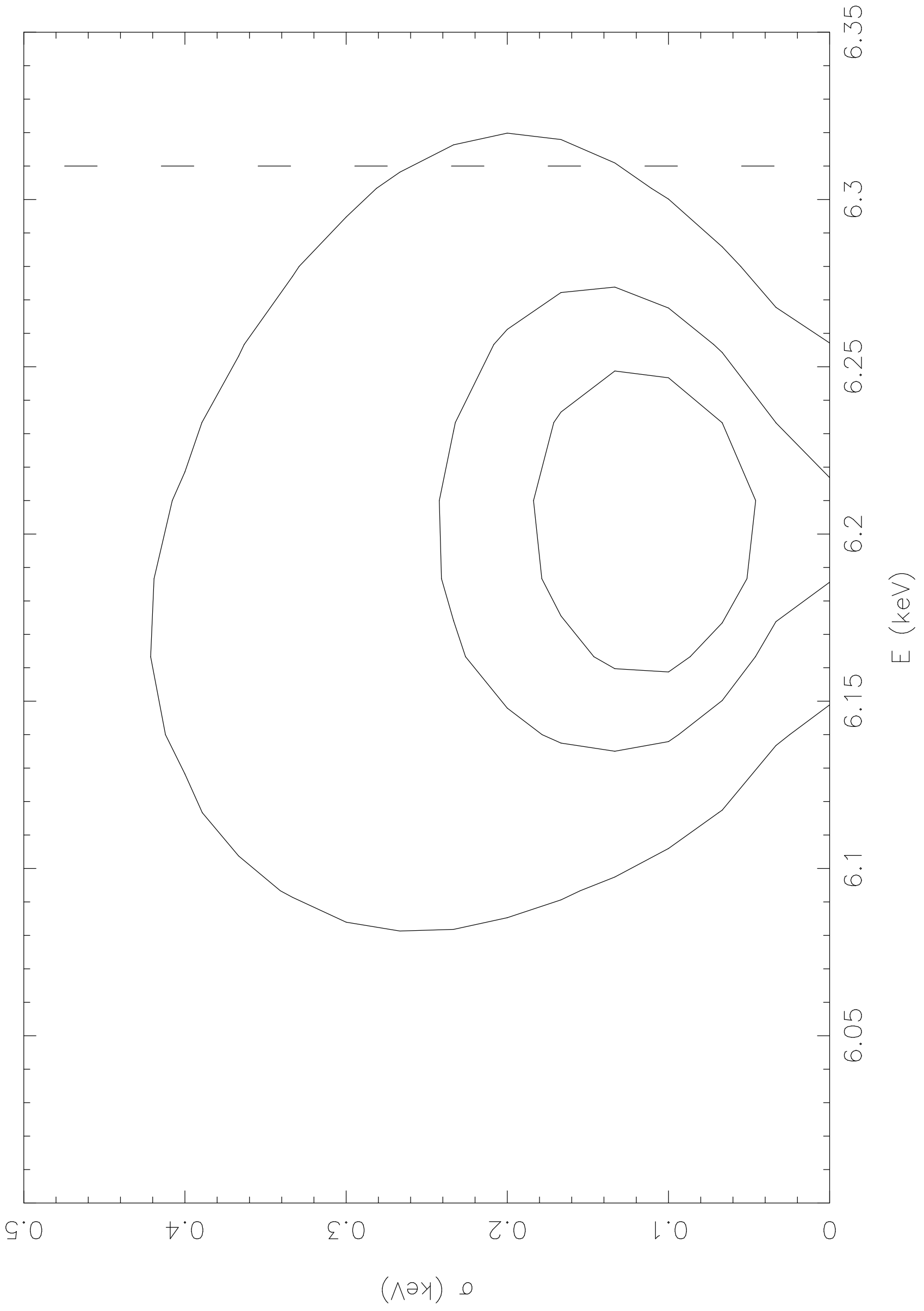




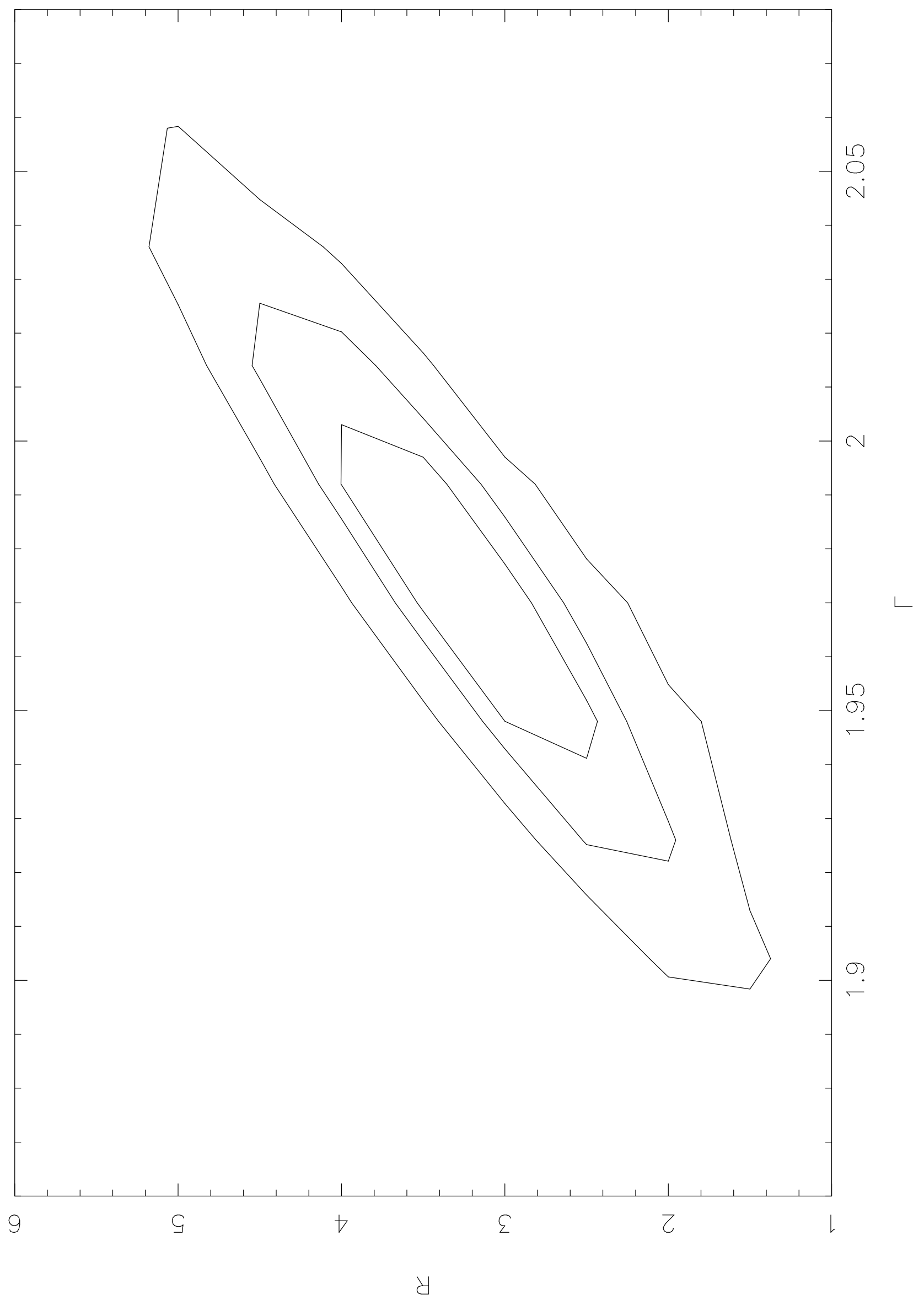




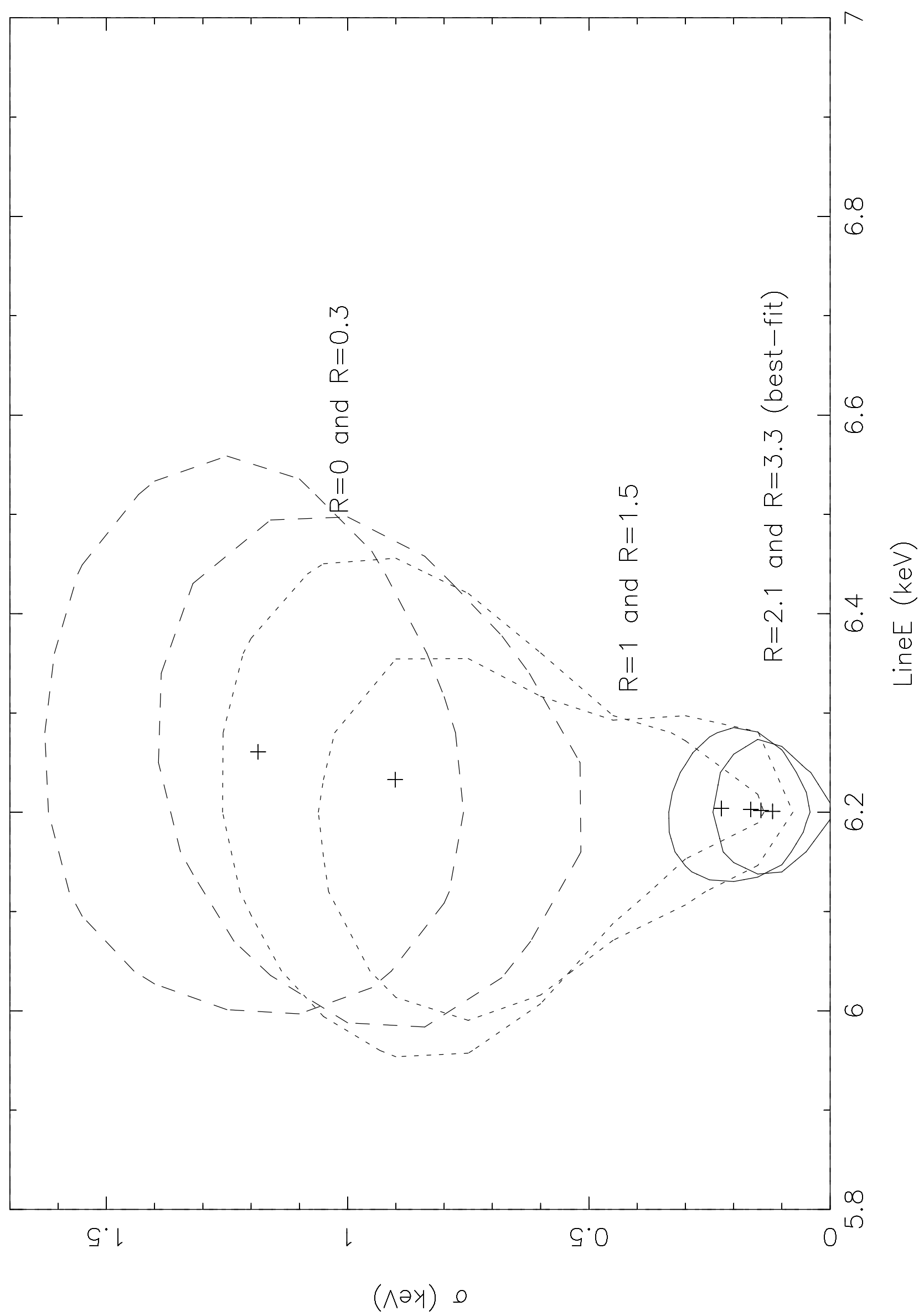


TABLE 3 - SIS0+1 Data $(0.4-10 \mathrm{keV})$

Results of Two Edges + Power Law + Fe K Line + Reflection Fits

\begin{tabular}{cccccccccccccc}
\hline \hline $\mathrm{E}_{1}$ & $\tau_{1}$ & $\mathrm{E}_{2}$ & $\tau_{2}$ & $N_{\mathrm{H}}$ & $\mathrm{T}$ & $\mathrm{A}_{\text {refl }}^{a}$ & $\mathrm{R}^{b}$ & $\mathrm{E}(\mathrm{FeK})$ & $\sigma(\mathrm{FeK})$ & $\mathrm{EW}(\mathrm{FeK})$ & $\chi_{\text {red }}^{2} /$ d.o.f. \\
$(\mathrm{keV})$ & & $(\mathrm{keV})$ & & $\left(10^{21} \mathrm{~cm}^{-2}\right)$ & & & & $(\mathrm{keV})$ & $(\mathrm{keV})$ & $(\mathrm{eV})$ & \\
\hline $0.70_{-0.02}^{+0.01}$ & $0.67_{-0.09}^{+0.07}$ & $0.84_{-0.02}^{+0.01}$ & $0.40_{-0.07}^{+0.07}$ & $3.48_{-0.13}^{+0.12}$ & $1.98_{-0.05}^{+0.05}$ & $102_{-34}^{+41}$ & $3.355_{-1.10}^{+1.17}$ & $6.20_{-0.06}^{+0.07}$ & $0.11_{-0.09}^{+0.07}$ & $89_{-32}^{+34}$ & $1.15 / 546$ \\
\hline \hline
\end{tabular}

Note: Intervals are at $90 \%$ confidence for one interesting parameter.

${ }^{a}$ Normalization in $10^{-3}$ photons $\mathrm{cm}^{-2} \mathrm{~s}^{-1} \mathrm{keV}^{-1}$ at $1 \mathrm{keV}$.

${ }^{b} \mathrm{R} \equiv \mathrm{A}_{\mathrm{refl} .} / \mathrm{A}_{\mathrm{pl}}$ (see section 3.2 for details) 